\title{
How do we conquer the recurrence of $\mathrm{HCC}$ ?
}

\author{
Kazuhiro Nouso
}

Received: 25 September 2014/ Accepted: 1 October 2014/Published online: 21 October 2014

(C) Springer Japan 2014

According to recent advancements in imaging modalities and due to the prevalence of surveillance systems for hepatocellular carcinoma ( $\mathrm{HCC}$ ), early detection of $\mathrm{HCC}$ has been achieved, and the proportion of patients who can receive local ablation therapy or surgical resection is increasing. Although tumors seem to be treated completely, recurrence of $\mathrm{HCC}$ has been frequently observed at an annual incidence rate of approximately $20 \%$. To achieve long survival of patients with HCC, suppression of recurrence is mandatory.

There are three conceivable strategies for coping with this high-hurdle problem. The first strategy is virus eradication. Hepatitis virus kills hepatocytes, and subsequently as cells regenerate replication errors of DNA occur, leading to hepatocarcinogenesis. The anti-carcinogenic effect of interferon and nucleotide analogs is well described, and their preventive effects against HCC development have been proven, especially for initial HCC occurrence. Virus eradication can abort this carcinogenic process from the starting point; however, it takes time to achieve a state free of recurrence because hepatocytes have already been injured before the use of these drugs is initiated, and some hepatocytes have already gained cancer potential.

The second strategy is prevention of the recurrence using anticancer drugs; however, this effect has been dismal. The survival of patients who have received uraciltegaful (UFT) after curative resection of HCC has not been

This comment refers to the article available at doi:10.1007/s00535-014-0996-1.

K. Nouso $(\square)$

Department of Gastroenterology and Hepatology, Okayama

University Graduate School of Medicine, Dentistry, and

Pharmaceutical Sciences, Okayama, Japan

e-mail: nouso@cc.okayama-u.ac.jp better than that of those who did not receive UFT as adjuvant therapy [1]. In addition, sorafenib did not reduce the recurrence rate after curative treatment in the STORM trial, a large, global, randomized controlled study (http:// clinicaltrials.gov.com, NCT00692770). These results indicate the difficulties of preventing recurrence using anticancer drugs.

The third strategy is to induce the differentiation of cancer cells. In this issue, Okita et al. present the result of a follow-up study that was originally conducted to verify the prophylactic effect of peretinoin for the prevention of HCC recurrence $[2,3]$. They report a promising survival benefit resulting from the use of peretinoin, especially for the patients with good liver function, Child-Pugh A. Peretinoin, an acyclic retinoid, is known to induce the transcriptional activation of the retinoic acid receptor and retinoid $\mathrm{X}$ receptor and to suppress tumor growth by inducing apoptosis and differentiation. This effect was observed only when $600 \mathrm{mg} /$ day of peretinoin was used. Unfortunately and unexpectedly, $300 \mathrm{mg} /$ day of peretinoin did not change the gene expression profile of cancer cells effectively [4], did not prolong recurrence-free survival in the original study, and did not prolong survival in this follow-up study. Due to the lack of beneficial effect at $300 \mathrm{mg} /$ day, peretinoin is still an investigational drug, and the confirmatory phase III study at $600 \mathrm{mg} / \mathrm{day}$ is in progress (http://clin icaltrials.gov.com, NCT01640808).

Almost 2 decades have passed since Muto et al. [5] reported that acyclic retinoid can prevent second primary HCC. The long road to proving the benefit of vitamin A analogs is approaching the end. Perhaps in the near future we will be able to obtain a new strategy: peretinoin for the prevention of recurrence and prolonging the survival of patients with early HCC. There is no doubt that the increased use of antiviral therapy for HCC patients will 
reduce the recurrence rate. By using antiviral drugs and peretinoin simultaneously, a synergistic effect is expected because they act via two different mechanisms. Consequently, results from this study shed light on the possibility of a complete cure of HCC. Whether an additional effect can be obtained by longer administration of peretinoin is the next question that needs to be answered.

\section{References}

1. Hasegawa K, Takayama T, Ijichi M, Matsuyama Y, Imamura H, Sano K, et al. Uracil-tegafur as an adjuvant for hepatocellular carcinoma: a randomized trial. Hepatology. 2006;44(4):891-5 (Epub 2006/09/29).

2. Okita K, Izumi N, Matsui O, Tanaka K, Kaneko S, Moriwaki H, et al. Peretinoin after curative therapy of hepatitis C-related hepatocellular carcinoma: a randomized double-blind placebocontrolled study. J Gastroenterol. 2014. doi:10.1007/s00535-0140956-9.

3. Okita K, Izumi N, Ikeda K, Osaki Y, Numata K, Ikeda M, et al. Survey of survival among patients with hepatitis $\mathrm{C}$ virus-related hepatocellular carcinoma treated with peretinoin, an acyclic retinoid, after the completion of a randomized, placebo-controlled trial. J Gastroenterol. 2014. doi:10.1007/s00535-014-0996-1.

4. Honda M, Yamashita T, Yamashita T, Arai K, Sakai Y, Sakai A, et al. Peretinoin, an acyclic retinoid, improves the hepatic gene signature of chronic hepatitis $\mathrm{C}$ following curative therapy of hepatocellular carcinoma. BMC Cancer. 2013;13:191 (Epub 2013/04/17).

5. Muto Y, Moriwaki H, Ninomiya M, Adachi S, Saito A, Takasaki KT, et al. Prevention of second primary tumors by an acyclic retinoid, polyprenoic acid, in patients with hepatocellular carcinoma. Hepatoma prevention study group. New Engl J Med. 1996;334(24):1561-7 (Epub 1996/06/13). 